\title{
Efectividad de un programa educativo sobre VIH/Sida aplicado a universitarios dentro del enfoque promocional
}

\author{
María Paulina Tello Delgado, ${ }^{\text {a,b,c }}$ Martha Edith Campos Santa Cruz. ${ }^{\text {a,d }}$
}

\begin{abstract}
Tello DMP, Campos SCME. Efectividad de un programa educativo sobre VIH/Sida aplicado a universitarios dentro del enfoque promocional. Cuid salud, ene-jun 2015; 2(1):125-136.
\end{abstract}

\section{RESUMEN}

Objetivo: determinar la efectividad del programa educativo sobre VIH/SIDA en universitarios tomando como referencia el modelo de promoción de la salud de Nola Pender y la teoría de autocuidado de Dorothea Orem. Metodología: estudio cuantitativo, pre experimental, con medidas de pre y pos test, realizado en una universidad privada, con una muestra de 60 estudiantes seleccionados por conveniencia según criterios pedagógicos e inclusión. El instrumento fue un cuestionario de 20 preguntas validado por juicio de expertos y prueba piloto, valor máximo 50 puntos, nota aprobatoria 14 en escala vigesimal. Los niveles de conocimiento alto, medio, bajo y muy bajo determinados por escala de estaninos; en el análisis se usó estadística descriptiva: media, desviación estándar. La efectividad se determinó con estadístico $\mathrm{X}^{2}$ con el método de McNemar nivel significación 1\% $(\alpha 0,01)$ Resultados: Antes de la intervención educativa el nivel de conocimiento fue muy bajo; después de la intervención se logró niveles de medio a alto, con promedio ponderado de 16,6. Conclusión: Se acepta la $\mathrm{H}_{1} p=0,005$, con un margen de error de $1 \%$, existen evidencias suficientes para afirmar que la aplicación del programa educativo sobre VIH/Sida incrementó significativamente el nivel de conocimiento en los universitarios participantes.

Palabras clave: conocimientos, educación en salud, VIH/Sida.
Tello DMP, Campos SCME. Effectiveness of an educational program on HIV/AIDS applied to university in approach promotional. Cuid salud, enejun 2015; 2(1):125-136.

\begin{abstract}
Objective: To determine the effectiveness of the educational program on HIV/AIDS in university reference to the promotion model of Nola Pender's health and self-care theory of Dorothea Oren. Methodology: quantitative study, experimental pre, measuring pre and post test, performed at a private university, with a sample of 60 students selected by convenience according to educational criteria and inclusion. The instrument was a questionnaire of 20 questions validated by expert judgment and pilot, maximum 50 points, 14 in 0 20 scale. Knowledge levels high, medium, low and very low was determined by estaninos scale; in the analysis used descriptive statistics: mean, standard deviation; The effectiveness was determined using the statistical method $\mathrm{X}^{2}$ McNemar $1 \%$ significance level ( $\alpha$ 0.01) Results: Before the educational intervention the level of knowledge was very low; after the intervention of medium to high levels was achieved, with a weighted average of 16.6. Conclusion: H1 $p=0.005$ are accepted, with a margin of error of $1 \%$, there is sufficient evidence to state that the application of the educational program on HIV / AIDS significantly increased the level of knowledge in college students participating.
\end{abstract}

Keywords: Knowledge, health education and HIV/AIDS.

\footnotetext{
${ }^{a}$ Enfermera Especialista en Docencia en Enfermería.

${ }^{\mathrm{b}}$ Enfermera Asistencial del Instituto Nacional de Salud del Niño.

${ }^{c}$ Docente de la Escuela de Enfermería Padre Luis Tezza, afiliada a la Universidad Ricardo Palma, Lima-Perú.

${ }^{\mathrm{d}}$ Enfermera Asistencial del Hospital San Bartolomé, Lima-Perú.
} 


\section{INTRODUCCIÓN}

La Organización Mundial de la Salud (OMS) considera que el Virus de Inmunodeficiencia Adquirida/Síndrome de Inmunodeficiencia Adquirida (VIH/Sida) sigue siendo un importante problema de salud pública mundial. ${ }^{1}$

Desde 1981, en que se inicia la pandemia, cerca de 75 millones de personas han contraído la infección por el virus. ${ }^{2}$ Si bien, entre el 2001-2012 los nuevos casos de infecciones por el VIH en adultos y jóvenes descendió aproximadamente en cincuenta por ciento en 26 países, con una prevalencia mundial de $0,3 \%$ en la población de 15-49 años y de 0,4\% en América Latina; ${ }^{2}$ este descenso ha sido desigual por regiones $\mathrm{y}$ poblaciones clave, dado que la epidemia se concentra en poblaciones con riego particular, como el caso de hombres que tienen sexo con hombres (HSH) y las mujeres trans (MTS); ambos grupos muestran un incremento mayor y ocupan los primeros lugares cuanto a porcentajes. ${ }^{3}$

En este contexto, todos los países reconocen que la población de jóvenes es un grupo de alta vulnerabilidad, existiendo preocupación por el incremento del comportamiento sexual de riesgo. Por otra parte, las estrategias y acciones preventivas de la epidemia son dirigidas hacia la población general, principalmente hacia el control de las infecciones de trasmisión sexual (ITS), diagnóstico de VIH y provisión de condones; los cuales se desarrollan en los centros de salud de nivel primario, reconociéndose la limitada oferta de servicios y de acceso que presentan estas entidades. ${ }^{3}$

En los últimos tiempos, la infección con el VIH en jóvenes entre 15 y 24 años ocurre con mayor frecuencia que antes, siendo que la mujer tiene mayor vulnerabilidad de adquirirlo por las propias desigualdades de género, ${ }^{4}$ con las implicancias que esto trae para sí misma y para la sociedad. Destacando que las muertes relacionadas con el Sida entre la población de 10-19 años aumentaron en 50\% entre el 2005-2012, en contraste a los progresos en la disminución de transmisión de madre-hijo. ${ }^{5}$

Entre los factores de transmisión del VIH en América Latina se encuentran el sexo heterosexual sin protección, el sexo entre hombres (HSH), el uso de drogas inyectables y, el trabajo sexual. ${ }^{6}$ Otro aspecto que incide en el aumento de casos en todo el mundo es que, chicas y chicos declaran haber tenido relaciones sexuales con personas contactadas a través de internet, ${ }^{7}$ medio que actualmente tiene gran impacto en la población de jóvenes, dado su fácil acceso a los avances tecnológicos.

A finales de 2011, 34 millones de personas vivían con el VIH en todo el mundo; calculándose que el $0,8 \%$ de adultos con edades de 15-49 años era seropositivo, aunque la morbilidad por la epidemia varía considerablemente entre países y regiones. ${ }^{8}$

En el Perú, un estudio realizado en los noventa identificó diferencia en la edad del inicio de las relaciones coitales heterosexuales en varones y mujeres, deduciéndose que este déficit de parejas femeninas estables al iniciar la vida sexual de los varones debía ser cubierto por personas con alto riesgo para la transmisión del VIH. Si estos se infectaban expondrían luego a sus parejas femeninas estables, desapareciendo el efecto protector de la monogamia en las mujeres. Los autores también identificaron una baja prevalencia del uso de condón y que los homosexuales, paralelamente, practicaban coito heterosexual y con mayor predisposición a practicar el sodomismo, constituyéndose así en transmisores potenciales del VIH y que solo una quinta parte de los varones sexualmente activos estaba exento de factores de riesgo conocidos. ${ }^{9} \mathrm{Al}$ respecto, el Director del sector salud de Cajamarca en el 2009 refirió en conferencia de prensa que: ${ }^{10}$

"[...] inicio de la epidemia de VIH en el Perú, el grupo etéreo más afectado fue el de los mayores de 30 años; sin embargo, con el correr de los años, la infección se ha presentado cada vez en gente más joven, de manera que la mayor proporción de infectados está entre 15 a 30 años[...]".

El primer caso de Sida en el país fue detectado por Patrucco en 1983. Luego, en 1985 se estarían detectando nueve casos más y, entre ellos, ocho ocurrieron en homosexuales y otro en hemofílico infectado por transfusión sanguínea, cuyas edades fluctuaban entre los 26-65 años. ${ }^{11}$

Actualmente, el Ministerio de Salud (MINSA) reporta que la mayor proporción de infectados se encuentran entre los 20-34 años en varones, mientras que en las mujeres el mayor número de casos ocurre en los de 25-34 años y de 45-49 años; además que los HSH registran el 56\% de los casos, ${ }^{12}$ destacando que entre los $\mathrm{HSH} /$ trans 
(+) solo se diagnostican el 24\%. ${ }^{3}$ Asimismo, señala que el $97 \%$ de casos de trasmisión de la enfermedad en el Perú ocurre por vía sexual.

Hasta enero del 2014, la Dirección General de Epidemiología (DGE) había reportado 31157 casos de Sida y 52490 VIH (acumulado); observándose poca variación a través de los años, pero con incremento en el riesgo. ${ }^{12}$ Existen estudios que señalan la precocidad de la vida sexual activa en los/las adolescentes, mostrando que mujeres y varones se inician antes de los 15 años, ${ }^{13-15}$ muchas veces sin la debida protección, agregándose a esto las elevadas tasas de ITS y la alta probabilidad de adquirir el VIH. ${ }^{16}$

A nivel mundial, ONUSIDA ${ }^{17}$ reporta que el $80 \%$ de las nuevas infecciones de VIH son transmitidas sexualmente; porcentaje que se incrementa al $97 \%$ en el Perú. En ese sentido esta institución señala:

"El Perú es un caso que merece destacarse: en una encuesta entre varones y mujeres jóvenes (1829 años), el 9\% de los varones indicó que al menos una de sus tres últimas parejas sexuales había sido un varón, y no habían utilizado preservativos en el $70 \%$ de estos contacto".p.2

Otro factor para el incremento de los casos de VIH/Sida en los jóvenes es el desconocimiento que tienen sobre la enfermedad. En el país, la mayoría de adolescentes varones no conoce suficientemente sobre las ITS y el VIH/Sida y muestra actitudes desfavorables frente al problema, ${ }^{18}$ hecho que aumenta el riesgo de contraer el VIH. Un estudio identificó que el $11,8 \%$ de varones y $15,3 \%$ de mujeres tienen conductas sexuales de alto riesgo; ${ }^{19}$ semejante a los resultados obtenidos en otras latitudes del mundo, tal y como lo señala Velandia, ${ }^{20}$ cuyo trabajo fue destacado en los diarios $\mathrm{ABC}^{21} \mathrm{y}$ Mediterráneo $^{22}$ en enero del 2015:

"[...] alerta del poco conocimiento en salud reproductiva y sexualidad de los alumnos de los primeros cursos, por la escasa formación recibida en la etapa educativa anterior, lo que aumenta el riesgo de enfermedades de transmisión sexual (ETS) y embarazos no deseados [...]. No hay una materia específica y obligatoria en colegios $e$ institutos, lo que provoca que más de la mitad de los estudiantes que llegan a la universidad "nunca han tenido una charla" sobre sexualidad y, simplemente, "tienen conocimientos básicos de genitales y sistemas reproductivos" [...]”. p. 27, 37.
Afirmaciones que confirman la escasez de conocimiento y la necesidad de educar a los jóvenes reconociendo sus derechos sexuales, así como la toma de conciencia sobre el panorama del VIH/Sida; resaltando la eficacia de las acciones de educación y promoción de la salud, especialmente de la salud sexual y reproductiva. Estudios orientados a elevar el conocimiento de los jóvenes plantean algunas estrategias que pueden ser de utilidad; entre ellos el modelo educativoparticipativo realizado en Cuba, que consiste en otorgar mayor libertad y autonomía al estudiante en la toma de decisiones. ${ }^{23}$ Un estudio de revisión sobre estrategias educativas destaca que la educación, la consejería y las campañas, además de la disponibilidad de manuales de capacitación constituyen medios de apoyo esenciales en este grupo; siendo requerido utilizar también técnicas de reflexión y análisis, dado que los jóvenes son sensibles a la opinión de sus pares y tienen influencia de sus compañeros. $^{24,25}$

Bajo este contexto, es indispensable la formación integral de los jóvenes en el tema sexual basada en el desarrollo humano, es decir, en el "ser" para así poder desarrollarse como personas y como seres humanos en interacción con los otros, ofreciendo herramientas que les ayuden a tomar decisiones con conocimiento de causa. En ese sentido, la educación no solo debe verse como un acumulo de conocimientos, sino como una formación para la vida. Esto hace evidente el desarrollo de una cultura de salud entre los jóvenes, ya que la educación y la salud no pueden estar desligadas; entendiendo que la salud es una fuente de felicidad y los universitarios necesitan estar en equilibrio y buen estado de funcionamiento en todas sus dimensiones humanas, de tal manera que su organismo pueda reaccionar eficientemente a los estímulos del mundo actual, siendo relevante proponer e innovar estrategias de cuidado orientados a ayudarles a vivir una vida sana y productiva.

Es importante reconocer la salud física y mental de los jóvenes como elemento básico en el progreso de un país. Principalmente cuando la enfermedad tiene consecuencias relevantes en su salud y desarrollo como es el caso del VIH/Sida, considerando que la mitad de infectados en el Perú se encuentran en la etapa de mayor productividad y que el mayor contagio ocurre por vía sexual; dado que los jóvenes tienden a experimentar con formas peligrosas de comportamiento, a menudo sin darse cuenta del peligro. 
La finalidad de este estudio fue desarrollar un modelo de intervención educativa sobre el VIH/Sida basado en el referencial de Nola Pender $^{26}$ y de Dorothea Orem. ${ }^{27}$ La primera autora propone el "modelo de promoción de la salud" y reconoce que los factores cognitivos y preceptúales son los principales determinantes de la conducta de promoción de la salud en el joven, es decir, las opciones que dispone, las características de la demanda y los rasgos estéticos del entorno en dónde se propone desarrollar ciertas conductas, como las acciones de protección de las ITS evitando grupos de riesgo. Desde esta teoría la balanza de los beneficios/barreras percibidos por el joven es relevante para continuar con prácticas de conductas saludables. Del Modelo Conceptual de Orem se tomó la teoría de sistemas de enfermería, específicamente el de "sistema de apoyo educativo". De este modo, la temática del VIH/Sida fue desarrollada objetivando afianzar las competencias y habilidades conceptuales, sociales y humanas que ayude a los jóvenes a tomar decisiones, mediante una actitud de reflexión y análisis. Ambas perspectivas confluyen en fortalecer el desarrollo de una vida sexual sana en la perspectiva de modificar conductas desfavorables para la salud y la vida.

\section{METODOLOGÍA}

Estudio cuantitativo, pre experimental, con medidas de pretest y postest. El área de estudio fue una universidad privada localizada en el norte del Perú, que cuenta con 21 escuelas profesionales y tiene una población estudiantil total de 7 475. Para el estudio se consideró una población de 150 universitarios congregados de forma accidental en el auditorio de la universidad; siendo elegidos 60 estudiantes de las diferentes carreras profesionales según criterios de inclusión: ambos sexos, participación voluntaria expresada en la firma del consentimiento informado y disponibilidad para acompañar el estudio de principio a fin, excluyéndose a los estudiantes de ciencias de las salud.

Para la ejecución del estudio, se cumplió previamente con los trámites administrativos, siendo la dirección de la escuela de enfermería intermediario; dado que la temática de VIH/Sida era parte de la asignatura de "educación en enfermería" y el programa educativo se incluyó en el curso, a modo de proyección social en el sexto ciclo académico.

La intervención educativa fue desarrollado por las investigadoras en dos días consecutivos de
08.00-12.00 horas en agosto del 2014, bajo el enfoque pedagógico socio crítico; se trabajó con grupos de 6 estudiantes, en la intención de motivarlos e integrarlos. Los temas abordados fueron: ITS, efectos del VIH sobre el sistema inmunológico, consejería, medidas de prevención, tratamiento y qué hacer para mantener latente el virus en caso de adquirirla, además de erradicar mitos sobre la enfermedad. De tal manera que los jóvenes pudieran internalizar conceptos y adoptar opciones saludables asumiendo responsablemente su sexualidad.

Para evaluar el nivel de conocimientos sobre VIH/Sida se aplicó un cuestionario de 20 preguntas (antes y después de la intervención educativa), sobre un total de 50 puntos: 15 preguntas sobre conceptos básicos generales de VIH/Sida, valor 2 puntos cada una y 5 preguntas sobre conocimientos de medidas de prevención y autocuidado, valor 4 puntos cada una. Se tomó en cuenta el criterio pedagógico de nota mínima aprobatoria 14, criterio utilizado en la universidad.

En la ejecución de la intervención educativa, la teoría de Pender brindó soporte para comprender cómo las interacciones influyen en los cambios de conducta, analizando críticamente el pensar, sentir, principios y valores, al mismo tiempo de trabajar con el fortalecimiento de la autoestima, modificar actitudes y prácticas relacionadas con la sexualidad y la prevención del VIH/Sida.

Este componente fue reforzado con la teoría de autocuidado de Orem, que tiene que ver con las decisiones y acciones de los jóvenes en beneficio de su propia salud, evaluando las barreras/beneficios presentes en el contexto de una vida sana y sin riesgos. Sobre esos elementos, haciendo uso del sistema de enfermería, componente activo del proceso de educación implementado por los investigadores, se dieron las pautas a los jóvenes, desarrollando los contenidos, evaluando cada necesidad individual y grupal, además de proyectar acciones de autocuidado a futuro; siendo de gran utilidad las dinámicas participativas, exposición diálogo y los relatos de experiencias, contando con el apoyo de multimedia y video.

Al iniciar la primera sesión el método de indagación fue primordial, partiéndose de la dinámica "trasmisión de la cadena" (se explica la dinámica, pero no se dice el objetivo, hasta que se establecen los grupos): tres jóvenes se visten de 
colores (verde, amarillo y rojo) portando diversas cintas del color de su vestimenta y eligen una cinta para tomarla, entregando el resto de cintas a tres personas más, quienes repiten el proceso y así sucesivamente; se trata de una competencia de quien entrega más cintas. Todos llegan a tener una cinta del color que desean, luego se forman grupos por el color de las cintas y permanecen hasta el final. Se da a conocer el significado de cada color (grupo rojo representa personas con VIH/Sida, amarillo personas con ITS y verde personas sanas); en este momento se indaga conocimientos sobre su salud, el cuidado de su cuerpo ¿cómo explican su sexualidad?, ¿Qué sentimientos, emociones y valores demuestran en relación al VIH/Sida; luego se trabajó la concientización, sensibilización, socialización y análisis del problema.

El objetivo era lograr participación activa y vivencial de los/las jóvenes, hecho que resulta ventajoso, pues los pares se entienden entre ellos, fomentándose aquí valores de respeto e identidad de género. Después de esto, se inició la exposición del tema, fomentando un dialogo constante con los jóvenes para compartir opiniones, experiencias, testimonios, evaluaciones, entre otros; explorándose en este momento las creencias, actitudes de auto-suficiencia, sentimientos de miedo, indiferencia, rechazo ante la enfermedad, entre otros. En cuanto al término "sexualidad", el $100 \%$ de jóvenes lo definió como "acto sexual". Puntos que se tomaron en cuenta para el siguiente día de trabajo, a fin de afianzar el conocimiento reforzándolo con un video.

Para determinar el nivel de conocimiento de los participantes se utilizó la escala vigesimal. Las respuestas con las calificaciones, tanto del pretest como del postest fueron registradas, ordenadas, tabuladas y procesadas mediante Microsoft Excel 2010 haciendo uso de códigos. En el análisis se usó la estadística descriptiva: intervalos, porcentajes, desviación estándar, frecuencia y media expresada en promedio.

Para la interpretación de los niveles de alto medio y bajo y muy bajo se baremó con escala de estaninos en el programa SPS versión 20. Finalmente, la efectividad del programa educativo fue determinada mediante la prueba de $\mathrm{X}^{2}$ por tratarse de dos pruebas relacionadas (pretest $\mathrm{y}$ postest) del tipo de cambio de conocimiento sobre VIH/Sida. Las observaciones se hacen en escala nominal (incremento y no incremento). Se utilizó un nivel de significación de $1 \%(\alpha=0,01)$, con la finalidad de evitar errores de tipo I.

\section{RESULTADOS}

Gráfico 1. Conocimientos sobre conceptos básicos del VIH/Sida en universitarios según pretest $\mathrm{y}$ postest. Chiclayo, Mayo-Junio, 2014.

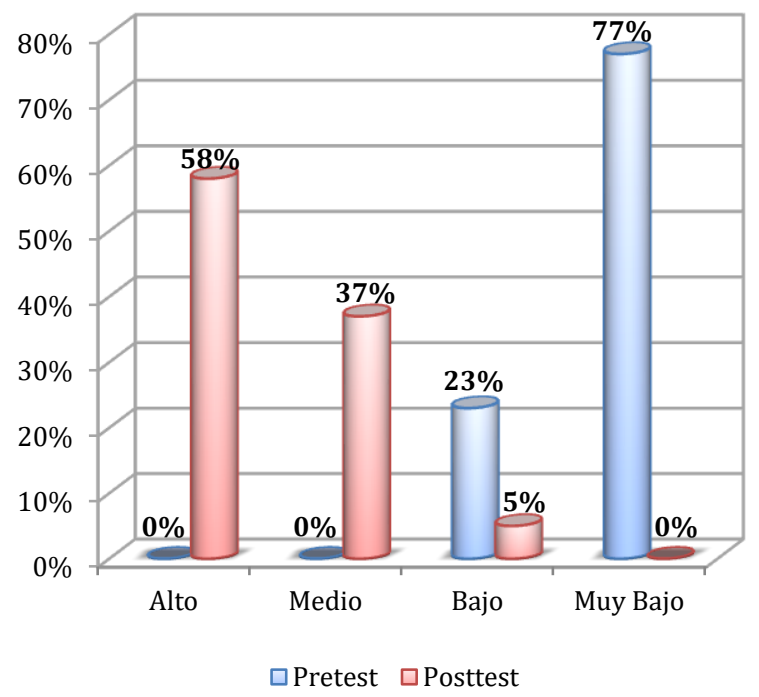

Gráfico 2. Conocimientos sobre medidas de prevención y autocuidado para evitar el VIH/Sida en universitarios, según pretest y postest. Chiclayo, Mayo-Junio 2014.

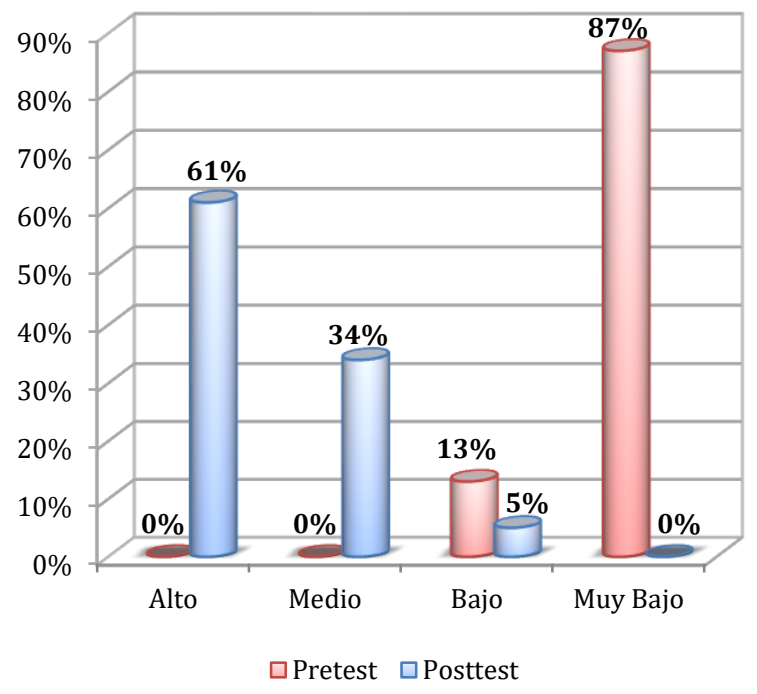

En el gráfico 1, en el pretest el 76,7\% (46) de universitarios alcanzó un nivel de conocimiento muy bajo y $23,3 \%$ (14) nivel bajo; resultados que se revirtieron en el postest, pues 58\% (35) alcanzó nivel alto y $38 \%$, (23) nivel medio.

En el gráfico 2, en el pretest el $87 \%$ (52) de universitarios alcanzó un nivel muy bajo de conocimiento y $13 \%$ nivel bajo. En el postest los resultados se invirtieron, pues el $61 \%$, (37) alcanzó un nivel de conocimiento alto y $5 \%$ (3) bajo. 
Gráfico 3. Conocimiento sobre medidas de prevención y autocuidado para evitar la trasmisión del VIH/Sida en universitarios en el pretest. Chiclayo. Mayo-Junio 2014

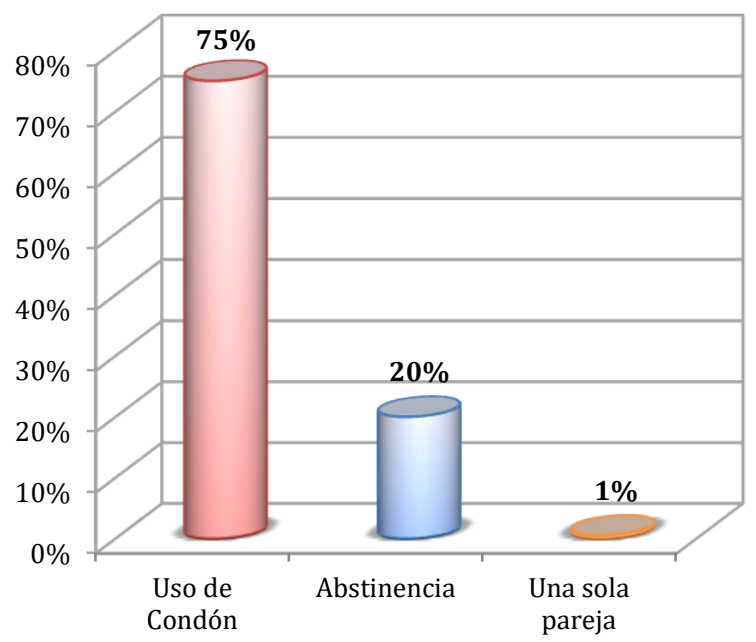

El gráfico 3, el 75\% (45) de universitarios reconoce el uso de condón como medida de prevención segura para evitar la trasmisión del VIH/Sida y solo el 5\% (3) indicó la monogamia, mientras que el 20\% (12) se inclinó por la abstinencia. Sin embargo, en datos no mostrados fue identificado que en la práctica el 60\% (36) de ellos usaba condón a veces, seguido de un $30 \%$ (18) que nunca lo usaba, frente a un $10 \%$ (6) que lo usaba siempre.

Al comparar la variable de estudio post intervención con los datos basales, se identifica diferencia significativa en el conocimiento $(p=0,005)$ entre los promedios del pretest $(10,9 \mathrm{de}$ calificación) y postest (16,6 de calificación). Denotando que al concluir el estudio los jóvenes manejaban mejor información sobre la protección contra el VIH/Sida.

\section{DISCUSIÓN}

La población universitaria está constituida por un alto porcentaje de jóvenes incluyendo los adolescentes, en el estudio el $90 \%$ de universitarios tenía entre 16-25 años de edad, con un promedio 19,2 $\pm 1,9$ de desviación estándar (DE); con una mayor proporción del sexo femenino. Cifras que se justifican porque el mayor porcentaje $(60 \%)$ de universitarios cursaba los primeros ciclos de estudios $\left(1^{\circ}-4^{\circ}\right)$.

La adolescencia y juventud son etapas vulnerables desde el punto de vista social, económico y de salud por sus estilos de vida desfavorables, el problema radica en que los jóvenes son impetuosos y no tienen percepción del riesgo, sobre todo la sociedad de hoy, porque están sometidos a una gigantesca publicidad que invita a la evasión, a la seducción y a la exposición al riesgo. Existen indicios preocupantes con respecto al incremento de los comportamientos sexuales de riesgo en los jóvenes; ${ }^{15-17}$ constituyendo un alerta para las autoridades sanitarias y los padres de familia, responsabilidad de la que no escapa el personal docente, tanto en la formación básica como universitaria.

Los resultados del estudio muestran un nivel de conocimiento sobre VIH/Sida muy bajo en los universitarios antes de la intervención (gráfico 1 y 2), corroborando hallazgos previos. ${ }^{18,20,21}$ Post intervención estos datos mejoran sustancialmente, siendo la menor calificación 13 (nivel bajo), nivel medio 14 (nota aprobatoria) y nivel medio-alto $16,2-16,6$.

En las medidas de protección y autocuidado, se identifica en los datos basales que solo el $10 \%$ de universitarios usa del condón siempre (gráfico 3), a pesar de ser reconocida como una medida segura de protección, lo que refleja desconocimiento o también inconciencia por parte de los jóvenes sobre el cuidado de su salud y la salud de los demás, atentando contra la salud pública. Al indagar durante la intervención el por qué no lo usaban, respondían que se olvidan, que no sienten placer, entre otros; siendo esto preocupante, ${ }_{2}$ dado su alta vulnerabilidad a adquirir el VIH o cualquier ITS al enfrentar su vida sexual sin responsabilidad o sí la tienen, desconocen cómo evitar el contagio, o pueden sentirse incapaces de comentar su uso con la pareja. Comportamiento reconocido por la OMS, ONUSIDA y el BM como uno de los factores responsables del incremento y prevalencia del virus. ${ }^{1-3}$

Aunque el Informe del MINSA, basado en datos del Fondo Mundial, reporta que los jóvenes mejoraron sus conocimientos sobre VIH y otras ITS, así como sus prácticas saludables, pero con menor efecto en el uso del condón. Avance que se ha detenido en los últimos años a causa del menor liderazgo del sector salud, de la reducción presupuetaria $\mathrm{y}$, escasa participación de la sociedad civil. ${ }^{28}$ Trabajo que parece haberse centralizado en la capital y las grandes urbes, Chiclayo es una ciudad donde aún falta afianzar el trabajo intersectorial, involucrando salud, educación básica y media, universidades, 
organismos no gubernamentales y la sociedad civil como un todo.

Sobre el uso del condón, se discutió y analizó arduamente durante la intervención educativa, a fin de concientizar a los jóvenes su importante función protectora no solo del embarazo, sino también del VIH e ITS. Resultado que se visualizó tempranamente en el postets, mientras que el $8 \%$ obtó por la abstinencia. Aunque esta respuesta no garantiza en sí misma un cambio comportamental. Estudios demuestran que las intervenciones para disminuir conductas de riesgo son efectivas a corto plazo, pero el reto es lograr que sean por periodos largos, ya que los jóvenes a pesar de contar con buen conocimiento sobre VIH/Sida no son sistemáticos en el uso de medidas de prevención, es decir, en muchos casos el conocimiento no se traduce en acción. ${ }^{24}$ De ahí que se propone capacitaciones y actividades educativas que refuercen la reflexión y el análisis de manera sostenible. ${ }^{25}$ Por esta razón, La educación sexual debe iniciarse desde muy temprano y de una manera conjunta por los padres y educadores.

El informe sobre prevención del VIH para adolescentes y mujeres jóvenes registra que los profesionales están insuficientemente preparados para abordar temas de sexualidad y del VIH. Situación semejante ocurre con los padres y docentes, quienes no han sido entrenados para abordar el tema de manera natural; destacando que en la cultura peruana no es común tratar estos temas abiertamente en clase o en la familia. ${ }^{29}$ Aun es necesario continuar el trabajo de capacitación y sensibilización con el personal docente de las escuelas de educación básica y media, actividad que debe ser continuada en el sistema universitario, acompañando el proceso de maduración de los jóvenes.

Durante la intervención educativa fue corroborado que los jóvenes desconocen sobre: su propia la anatomía, la consejería, a dónde acudir en caso de exponerse a una relación de riesgo, cómo conservar los condones (la mayoría indicó que los lleva en su billetera). Muy pocos conversan con sus padres y/o parejas sobre temas de sexualidad. Algunas respuestas identificadas en los universitarios fueron: "solo se enferman los promiscuos [...] cochinos [...], yo sé con quién me meto [...]”; "[...] en el supuesto caso de tener una prueba positiva al VIH, no haría nada, no le diría a nadie [...] por temor al rechazo[...]". Todas estas expresiones reflejan el poco valor que tiene la salud para los jóvenes, además de irresponsabilidad, inconciencia o debido a la ¿ignorancia?. En general, se perciben fuera del problema, por lo tanto no requieren cambiar sus conductas sexuales de riesgo, consideran que la enfermedad lo pueden adquirir otros pero no ellos, se creen invulnerables.

Para comenzar, existe confusión en la conceptualización del término "sexualidad" con el "acto sexual". La primera incluye más que solamente sexo, incorpora el mundo de las relaciones afectivas y sentimientos que construyen una actividad humana esencial. Según la OMS, citado por Velasquez, ${ }^{30}$ la sexualidad es un aspecto central del ser humano, presente a lo largo de la vida, abarca el sexo, las identidades, los roles de género, el erotismo, el placer, la intimidad, la reproducción y la orientación sexual.

Para Lázaro, ${ }^{31}$ la sexualidad saludable es la que produce bienestar físico, emocional $\mathrm{y}$ sentimental, en la que se puede disfrutar libremente con la pareja, es estar en un ambiente adecuado, tener comunicación $\mathrm{y}$, sobre todo, es estar informado para tomar las decisiones correctas $\mathrm{y} \sin$ miedo.

En ese sentido, la información provista a los jóvenes debe ser veraz, objetiva y sensible, en la que juega un importante papel la familia, los centros educativos y las universidades, que permita reflexión y análisis profundo sobre la importancia de la sexualidad en el curso de la vida y no solo en el momento. Los jóvenes necesitan tener cierto bagaje de conocimientos sobre el cuidado de su salud como herramienta de libre elección de conducta saludable y responsable, que no trasgreda la salud del otro (pareja).

De ello se desprende que la educación en salud es un elemento fundamental en la promoción de comportamientos, trabajar sobre el área cognitiva constituye el primer eslabon para modificar conductas y promover estilos de vida saludable en las personas. Los resultados muestran incremento significativo del conocimiento $(p=0,005)$ en los universitarios, similares a los datos obtenidos por Mariño y Santoya ${ }^{23}$ y Gonzáles et al., ${ }^{24}$ quienes promovieron una mayor libertad y autonomía en el estudiante para la toma de decisiones, como consejería, campañas, reflexión y análisis, además de proveer materiales como manuales.

Desde el enfoque de la promoción de la salud de Pender, la intervención educativa fue 
encaminada a desarrollar procesos y actividades con los jóvenes, de manera individual o grupal, con fin de modificar conductas de riesgo y adoptar estilos de vida saludables, mejorando su calidad, entendiendo que los jóvenes son entidades biopsicosociales y que están influenciables por el medio dónde se desarrollan. Aquí, cobra relevancia los procesos cognitivos para modificar los comportamientos, no solo desde la orientación sino desde una convivencia mutua que refuerza lo que se desea cambiar, pudiendo estar representado este apoyo en los padres, el personal de salud. ${ }^{26} \mathrm{El}$ programa sobre VIH/Sida fomentó el desarrollo de habilidades personales en los jóvenes para asumir la protección de la salud.

Se sabe que una persona bien informada tomará mejores decisiones en función de su propio bienestar, evitando o reduciendo los riesgos en un ambiente que induce a la liberación sexual, enajenación del otro, cosificación de la mujer. ONUSIDA informa que las personas adquieren la enfermedad en las primeras décadas de su vida (20-29 años) y que el diagnóstico generalmente ocurre entre la tercera o cuarta década de vida. ${ }^{17}$ Ante esta realidad, se cree que la educación sexual puede lograr en los jóvenes elecciones más seguras y responsables. Datos de la ENDES 2012, citado por el MINSA en el Informe Nacional sobre los progresos del VIH/Sida en el país, reporta que las mujeres con niveles educativos bajos y pertenecientes al quintil inferior de riqueza desconocen aspectos relacionados con el VIH/Sida. ${ }^{28}$

El modelo de promoción de la salud permitió establecer la interconexión que existe entre los factores cognitivos-perceptuales, experiencias, creencias y las ideas que los universitarios poseen respecto al VIH/Sida y su salud. Siendo los factores demográficos y socio-ambientales como la edad, género, pares, comunicación masiva, entre otros; elementos claves para analizar la problemática en cuestión. Dado que el ingreso a la universidad genera cambios en la vida de los jóvenes (como apartarse de la familia, del hogar, nuevas normas y adaptaciones, nuevos compañeros de estudio), generando altos niveles de estrés y manejo del tiempo; contexto en el que pueden asumir conductas saludables o de riesgo para su salud, dependiendo de las oportunidades que se presentan $y$ ante el cual es necesario ofrecer soporte, imágenes y modelos a seguir; lo que implica una cultura de estilos de vida saludables como responsabilidad en todo el claustro universitario.
A través del Plan Estratégico Multisectorial de VIH/Sida 2007-2011, citado por el MINSA, ${ }^{28}$ se han logrado avances en los aspectos preventivos promocionales especialmente al incrementar el conocimiento sobre VIH y otras ITS, mayor uso de condón en las poblaciones de 12-18 años y de 1924 años, aunque esta mejora no es uniforme en todo el país. La misma entidad informa que el manejo y prevención del VIH/Sida está dirigido prioritariamente a grupos altamente vulnerables como los HSH y los MTS, quedando los y las adolescentes de 15-24 años de edad fuera de este enfoque. $^{29}$

La enfermera trabajando en colaboración con las escuelas y universidades sería de gran apoyo, fomentando la adopción de estilos de vida saludables, partiendo desde sus experiencias y creencias, valores y expectativas; además de acompañarlos en el proceso de construcción, de la búsqueda de identidad, fortalecimiento $\mathrm{y}$ definición del carácter. Orem refiere que la salud es un estado altamente positivo y no simplemente la ausencia de la enfermedad. ${ }^{27}$ En ese sentido, sí los universitarios tienen el conocimiento suficiente sobre la importancia del autocuidado, de la prevención y promoción de la salud en cuestiones de vida sexual, sabrán analizar, internalizar y adoptar opciones saludables asumiendo un mayor control sobre sí mismos. Estudios revelan que la inclusión de la educación en salud en los programas de estudio escolar, universitario, mejora las aptitudes, el conocimiento y las intenciones conductuales de los jóvenes. $^{23,25}$

El rol de educador de la enfermera en la promoción de la salud de la población adolescente y adulto joven dentro del ámbito universitario es imperativo, pues trabajar por la salud de estos grupos etarios es trabajar por el desarrollo humano, social y económico del país. Especialmente porque se encuentran en una etapa del ciclo vital clave para la adopción de estilos de vida, que practicarán en el ámbito familiar, social y laboral, ${ }^{32}$ siempre y cuando los mismos se constituyan en soporte constante y en modelos de referencia.

No obstante, en la práctica su importancia para asegurar mejoras en la salud de la población en general y, sobre todo en los grupos vulnerables (en las que se incluyen los jóvenes), aun parece ser un caso aparte; las mallas curriculares universitarias, no incluyen la educación en salud de modo explicito como parte de la formación integral. Existen escazos programas de salud que reconocen las diversidades sexuales e identidades 
de género, a pesar de constituir grupos vulnerables al VIH/Sida (HSH, MTS).

Frente a este aparente vacio, las facultades y escuelas de enfermería pueden ser entes protagónicos de la promoción de la salud en sus espacios, desarrollando cursos de capacitación y talleres educativos para docentes; acompañamiento, asesoría, tutorías, formación de comités de salud en la comunidad estudiantil universitaria y comunal, a fin de generar consciencia de autocuidado y prácticas de estilos de vida saludable, sumándose a los objetivos establecidos en el Plan Multisectorial de VIH/Sida 2007-2011. Es necesario una mayor participación de los organismos universitarios, compete a todos buscar y estrechar lazos entre entidades intersectoriales, especialmente cuando se tiene la capacidad y los recursos humanos, específicamente quienes cuentan con Facultades de Ciencias de la Salud.

\section{Correspondencia:}

Maria Paulina Tello Delgado

Correo electrónico: matello2@hotmail.com

\section{REFERENCIAS BIBLIOGRÁFICAS}

1. Organización Mundial de la Salud (OMS): Centro de Prensa [internet]. Ginebra: OMS; c.s.f.-2015. Nota descriptiva $\mathrm{N}^{\circ} 360$. VIH/Sida; octubre 2013 [citado 10 ene 2015]; [aproximadamente 3 laudas]. Disponible en: http://www.who.int/mediacentre/factsheets/fs 360/es/

2. ONUSIDA. Informe mundial sobre la epidemia mundial de sida 2013 [internet] Geneva-Switzerland: ONUSIDA; 2013 [citado 29 Set 2014]. p.274. Disponible en: http://www.unaids.org/sites/default/files/medi a_asset/UNAIDS_Global_Report_2013_es_1 .pdf

3. ONUSIDA. Informe Mundial. Informe de ONUSIDA sobre la epidemia mundial de sida 2012 [Internet]. Ginebra-Suiza: ONUSIDA; 2012 [citado 21 de ago 2014]. p.212. Disponible en: http://www.unaids.org/sites/default/files/en/m edia/unaids/contentassets/documents/epidemi ology/2012/gr2012/20121120_UNAIDS_Glo bal_Report_2012_with_annexes_es.pdf
Finalizando, la intervención propuesta en el estudio fue efectiva en la mejora del conocimiento de los universitarios, siendo necesario conocer a posteriori cómo este conocimiento influye en las decisiones de la vida, si modifica y en qué medida las conductas de riesgos de los jóvenes (uso de condón, abstinencia), lo que demanda actividades de acompañamiento y tutoría a lo largo del tiempo para consolidar las buenas prácticas sexuales de protección.

Una de las limitaciones del estudio fue la autoselección de los universitarios para participar del programa, además de no contar con grupo de comparación, sugiriéndose replicar la experiencia en poblaciones de mayor tamaño, considerando estudios longitudinales prospectivos, a fin de corroborar la eficacia del modelo asumido en el programa sobre VIH/Sida y lograr la meta que se persigue en la promoción de la salud, cambios de comportamiento y un desarrollo de la sexualidad libre de riesgos.

4. Luciano D, Lacono M. Manual para fortalecer el ejercicio de los derechos humanos de las mujeres que viven con VIH en América Latina [internet]. Ciudad de Panamá: ONUSIDA, CIM/OEA; dic 2014 [citado 01 de ene 2015]. p.128. Disponible en:

http://www.oas.org/es/cim/docs/Manual_CI $\underline{\text { M_ONUSida_ES.pdf }}$

5. ONUSIDA/UNICEF. Declaración de prensa [internet]. Ginebra: ONUSIDA/UNICEF; s.f. [citado 10 de jul 2014]. p.3. Disponible en: http://www.unaids.org/sites/default/files/web story/20131129_PR_UNICEF-UNAIDSStocktaking-Report_es_0.pdf

6. Banco Mundial. Enfrentar el desafío: Proyectos y operaciones [Internet]. Washington DC: Banco Mundial; c.s.f.-2015. El Banco Mundial y el VIH/sida; abr de 2013 [citado 04 de jun 2014]; [aproximadamente 4 laudas]. Disponible en: http://www.bancomundial.org/es/results/2013 /04/03/hivaids-sector-results-profile 
7. Folch C, Álvarez JL, Casabona J, Brotons M, Castellsagué $\mathrm{X}$ et al. Determinantes de las conductas sexuales de riesgo en jóvenes de Cataluña. Rev Esp Salud Pública [Internet]. 2015 [citado 8 de may 2015]; 89(5): 471-85. Disponible en: http://www.msssi.gob.es/biblioPublic/publica ciones/recursos_propios/resp/revista_cdrom/v ol89/vol89 5/RS895C_CF.pdf

8. ONUSIDA. La epidemia de SIDA en América Latina y el Caribe [Internet]. Ginebra-Suiza: ONUSIDA; s.f. [citado 10 de ene 2015]. p. 2. Disponible en: http://data.unaids.org/publications/factsheets04/fs_lamerica_carib_es.pdf

8. Valdéz BH, Adachi MR, Gotuzzo HE. Prácticas sexuales en la población universitaria de Lima, 2013: Análisis de los factores de riesgo para la transmisión sexual del virus de la inmunodeficiencia humana. Rev Med Hered [serie de internet]. 1991 [citado 20 de abr 2014]; 2(1): [aproximadamente 10 laudas]. Disponible en: http://www.upch.edu.pe/vrinve/dugic/revistas lindex.php/RMH/article/view/298/265

9. Panorama Cajamarquino [Internet]. Cajamarca: Panorama Cajamarquino; c.s.f.2016. Cajamarca reporta 117 casos de VIHSIDA; dic 032009 [citado 26 de sep 2014]; [aproximadamente 01 lauda]. Disponible en: http://www.panoramacajamarquino.com/noti cia/cajamarca-reporta-117-casos-de-vih$\%$ E2\%80\%93-sida/

11. Cueto M. El rastro del SIDA en el Perú. Hist. cienc. saude-Manguinhos [serie de internet]. 2002 [citado 6 de ene 2015]; 9(Suppl): 17 40. Disponible en: http://www.scielo.br/pdf/hcsm/v9s0/01.pdf

12. Dirección General de Epidemiologia (DGE). Situación Del VIH/SIDA en el Perú. Boletín Epidemiológico Mensual [internet]. LimaPerú: MINSA/DGE; ene 2013 [citado 4 de may 2015]. p.01. Disponible en: http://www.dge.gob.pe/portal/docs/vigilancia/ vih/Boletin_2014/enero.pdf

13. Ministerio de Salud. Boletín Epidemiológico 2013. VIH/SIDA [Internet]. Lima-Perú: MINSA; ene 312014 [citado 23 de abr 2014]. p.02. Disponible en
http://www.rslc.gob.pe/Descargas/Epidemiol ogia/Boletin/2013/Boletin-N-12-2013.pdf.

14. Royuela RP, Rodríguez Molinero LB, Marugán MJM, Carbajosa RV. Factores de riesgo de la precocidad sexual en adolescentes. Rev Pediatr Aten Primaria [serie de internet]. 2015 [citado 11 de may 2015]; 17:127-36. Disponible en: http://www.pap.es/files/1116-1956pdf/RPAP 66 Factores_riesgo_sexual.espeng.pdf

15. Abreu NR, Oliva RA, García RGN, León JM, Naranjo LML. Adolescencia e inicio precoz de las relaciones sexuales. Algunos factores desencadenantes. Gaceta Médica Espirituana [serie de internet]. 2008 [citado 6 de ago 2014]; 10(2): [aproximadamente 5 laudas]. Disponible en: http://bvs.sld.cu/revistas/gme/pub/vol.10.(2) $\underline{01 / p 1 . h t m l}$

16. Parodi-Carro AF .Educación sexual y el perfil del adolescente de nivel secundario en San Juan de Lurigancho-Lima. Cuid Salud [serie de internet]. Jul-dic 2014 [citado 08 de ene 2015]; 1(2):67-76. Disponible en: http://eeplt.edu.pe/revista/index.php/Salud/art icle/view/21

17. ONUSIDA. La epidemia de SIDA en América Latina y el Caribe [internet]. Ginebra: ONUSIDA; 2011 [citado 4 de abr 2014]. p.2. Disponible en: http://data.unaids.org/publications/factsheets04/fs_lamerica_carib_es.pdf

18. Sánchez RVJ. Conocimientos y actitudes acerca de las ITS y el VIH/Sida de los adolescentes varones en dos comunidades zonales de Chimbote-Perú, 2010. Crescendo, ene.-jun 2011; 2(1):161-177.

19. Vásquez DSPM, Rojas GM, Torres SR, Salazar BN, Alvarado CH, Díaz MM, et al. Conocimientos en VIH/SIDA y comportamiento sexual en estudiantes de la UNAP. Iquitos 2009. Conocimiento [internet]. Ene-jun 2010 [citado 3 de jun 2014]; 9(1): 80-88. Disponible en: http://200.48.67.60/menus/archivos/Conocimi ento.

20. Velandia MMA. Estrategias docentes en enfermería para el abordaje de la sexualidad, 
salud sexual y salud reproductiva en adolescentes y jóvenes [tesis doctoral]. Alicante-España: Universidad de Alicante, 2011 [citado 20 de oct 2014]. p.370. Disponible en: http://es.scribd.com/doc/56943629/TesisDoctorado-Manuel-Antonio-VelandiaMora\#scribd

21. ABC. Salud [internet]. Alicante-España: ABC; c.s.f.-2015. Los universitarios saben poco de sexo; ene 24 de 2015 [citado 20 de may 2015]; [aproximadamente 3 laudas]. Disponible en: http://www.abc.es/localalicante/20150124/abci-universitariosestudio-sexo-201501241204.html

22. Mediterráneo. Sociedad [internet]. AlicanteEspaña: Mediterráneo; c.s.f.-2016. Alertan del desconocimiento de salud sexual de los universitarios; ene 25 de 2015 [citado 20 de may 2015]; [aproximadamente 3 laudas]. Disponible en: http://www.elperiodicomediterraneo.com/noti cias/sociedad/alertan-desconocimiento-salud$\underline{\text { sexual-universitarios_915631.html }}$

23. Mariño SRY, Santoya AAA. Intervención educativa sobre VIH/sida. Rev Hum Med [serie de internet]. Sep-dic 2013 [citado 10 de feb 2014]; 13(3): 622-38. Disponible en: http://scielo.sld.cu/scielo.php?script=sci_pdf \&pid $=$ S172781202013000300004\&lng=es\&nrm=iso\&tlng =es

24. García-Mora A, Mendoza-Brizio MA, González-Gutiérrez A, Hernández-González M, Ortega-Escamilla PG. Conocimientos y prácticas de riesgo ante VIH/SIDA en adolescentes de la facultad de planeación Urbana y Regional de la Universidad Autónoma del Estado de México. Revista de Medicina e Investigación [serie internet]; 2014 [citado el 10 may 2015], 2(2): 121-27. Disponible en: http://www.elsevier.es/enrevista-revista-medicina-e-investigacion-353articulo-conocimientos-practicas-riesgo-ante$\underline{\text { el-90360376 }}$

25. González VB, Bolaños GMR, Pupo ANL. Estrategias educativas para la prevención de las infecciones de trasmisión sexual en jóvenes. Rev cub salud pública [serie de internet]. Dic 2010 [citado 10 de sep 2014]; 36 (4): 295-300. Disponible en: http://www.scielosp.org/scielo.php?script=sci $\_$arttext\&pid=S0864-

34662010000400002\&lng=en.http://dx.doi.or $\mathrm{g} / 10.1590 / \mathrm{S} 0864-662010000400002$

26. Sakraida TJ. Nola J Pender. El modelo de promoción de la salud. En: Tomy AM, Alligood MR. Modelos y teorías en enfermería. 5ta edición, Madrid-España: Elservier Mosby; 2005. Capítulo 33, p.62540.

27. Taylor SG. Dotohea E Orem. La teoría enfermera del déficit de Autocuidado. En: Tomy AM, Alligood MR. Modelos y teorías en enfermería. 5ta edición, Madrid-España: Elservier Mosby; 2005. Capítulo 13, p.1892013.

28. International Planned Parenthood Federation (IPPF), Fondo de Población de las Naciones Unidad-Perú (UNFPA-Perú), The Global Coalition in Women and AIDS, Young Positives. Informe: Prevención del VIH para adolescentes y mujeres jóvenes [internet]. Lima-Perú: IPPF, UFPA-Perú; s.f. [citado 10 de may 2015]. p.8. Disponible en: /hiv_prevention_girls_and_young_women_p eru_report_card_spanis.pdf

29. Ministerio de Salud (MINSA). Informe nacional sobre los progresos realizados en el país. Perú. Periodo 2012-diciembre 2013 [internet]. Lima-Perú: MINSA; diciembre 2013 [citado 10 de ene 2015]. p.175. Disponible:

http://www.unaids.org/sites/default/files/en/d ataanalysis/knowyourresponse/countryprogre ssreports/2014countries/PER narrative_repor t 2014.pdf

30. Velásquez CS. Sexualidad Responsable. Unidad 2 [Internet]. México: Universidad Autónoma del Estado de Hidalgo, Sistema de Universidad Virtual; s.f. [citado 22 de jun 2014]. 12 p. Disponible en: http://www.uaeh.edu.mx/docencia/VI_Presen taciones/licenciatura en mercadotecnia/fund amentos_de_metodologia_investigacion/PRE $\underline{\text { S44.pdf }}$

31. Lázaro MMA. Sexualidad Saludable [home page]. Barcelona: Grupo de Trabajo sobre Tratamiento del VIH; oct 3 de 2009 [actualizado 10 de sep 2014; citado 15 de ene 
2015]. Disponible en: $\underline{\text { http://gtt- }}$ vih.org/cuidate/sexualidad_y_vih/sexualidad saludable

32. Morales IG, Valle RC, Soto VA, Ivanovic MD. Factores de riesgo cardiovascular en estudiantes universitarios. Rev Chil Nutr [serie de internet]. Dic 2013 [citado 18 de may 2015], 40(4): 391$396 . \quad$ Disponible en: http://www.scielo.cl/pdf/rchnut/v40n4/art10.p $\underline{\mathrm{df}}$ 